\title{
Network Influence based Classification and Comparison of Neurological Conditions
}

\author{
Ruaridh Clark $^{1}$, Niia Nikolova ${ }^{2}$, Malcolm Macdonald ${ }^{1}$, and \\ William J. McGeown ${ }^{2}$ \\ 1 Mechanical \& Aerospace Engineering, University of Strathclyde, Glasgow, UK \\ ruaridh.clark@strath.ac.uk \\ 2 School of Psychological Sciences and Health, University of Strathclyde, Glasgow, \\ UK
}

\begin{abstract}
Variations in the influence of brain regions are used to classify neurological conditions by identifying eigenvector-based communities in connectivity matrices, generated from resting state functional magnetic resonance imaging scans. These communities capture the network influence of each brain region, revealing that the subjects with Alzheimers disease (AD) have a significantly lower degree of variation in their most influential brain regions when compared with healthy control (HC) and amnestic mild cognitive impairment (aMCI) subjects. Classification of subjects based on their pattern of influential regions is demonstrated with neural networks identifying HC, aMCI and AD subjects. The difference between these conditions are investigated by altering brain region influence so that a neural network changes a subjects classification. This conversion is performed on healthy subjects changing to aMCI or $\mathrm{AD}$, and for aMCI subjects changing to AD. The results highlight potential compensatory mechanisms that increase or maintain functional connectivity in certain regions for those with aMCI, such as in the right parahippocampal gyrus and regions in the default mode network, but these same regions experience significant decline in those that convert from aMCI to AD.
\end{abstract}

Keywords: functional connectivity, community detection, dementia

\section{Introduction}

Alzheimers disease (AD) is the most common type of dementia. It is typically characterised by marked decline in episodic memory, with deficits occurring in other cognitive domains such as in language, visuospatial and executive functioning. Individuals with amnestic mild cognitive impairment (aMCI) present with impairments in memory, with other cognitive domains remaining relatively intact. Although not all individuals with aMCI are in the early stages of $\mathrm{AD}$, people with this pattern of symptomology are at high risk of conversion to the disorder [1].

There has been a large body of work documenting the changes in regional brain volume as the disease progresses, for example atrophy of the hippocampus 
is an indicator that subjects with aMCI will develop $\mathrm{AD}$ [2]. Whereas volumetric analysis does not inform on how brain dynamics are modified by $\mathrm{AD}$, functional magnetic resonance imaging (fMRI) data may offer information on the regulation of brain networks and provide additional markers of disease. This is an important distinction, as the volume of the parahippocampal gyri (PHG) has been found to remain constant for those with aMCI regardless of whether they declined further into $\mathrm{AD}$ or not, but a greater extent of activation within the PHG was a reliable marker of future cognitive decline [3].

This paper will focus on functional connectivity analyses to differentiate between people with AD and aMCI. The approach taken here is to identify and compare the relative influence of brain regions. This influence is captured from resting state fMRI data that is converted into a network of brain regions, with connections weighted by the strength of their signal correlations [4]. A region's influence is determined using an eigenvector-based community detection, communities of dynamical influence (CDI), as introduced by Clark, Punzo and Macdonald (2019) [5]. For a directed graph, this influence represents the nodes that can rapidly lead the network to a new state of consensus. For an undirected graph, used here to represent brain region connectivity, information is lost on which nodes are leading or following. Therefore, influential nodes are either important sources or sinks for information in the network. The CDI method relies on the relationship between eigenvectors to determine the communities, where the most dominant eigenvectors form a coordinate system with communities displaying as an alignment of nodes from that system's origin. Eigenvectors have been used previously with the neuronal network of the $C$. elegans to identify brain circuitry [6]. CDI is a progression from normalised cuts [7], and other spectral bisection methods, as it considers a combination of eigenvectors before determining community assignment.

By associating each brain region with an influence ranking, based on which community it belongs to, a subject can be characterised based on the relative influence of their brain regions. Pattern recognition enables the detection and association of these rankings with different neurological conditions. Machine learning (ML) is a highly capable and popular method of recognising statistical patterns, where most of the research on classifying aMCI and AD with ML has focused on low-level features such as cortical thickness and/or grey matter tissue volumes from MRI, mean signal intensities from positron emission tomography (PET) and other common biomarkers [8]. There are also examples of graph theoretical metrics being used in combination with machine learning, such as [9] \& [10] that employed a support vector machine (SVM) as well as [11] that used a naïve Bayes classifier to perform the classification. Of significant clinical utility, functional brain connectivity can be combined with machine learning into models capable of identifying neurological conditions such as $\mathrm{AD}$ [12], autism or depression.

In this paper, we describe a novel method of determining the influence of brain regions in neurological conditions, by identifying communities of dynamical influence in healthy controls, individuals with aMCI and patients with AD. We also determine how influence may change during conversion between these 
conditions, by developing a new technique to classify individuals based on the influence of their brain regions.

\section{Methods}

In order to classify and compare influential communities between the AD, aMCI and control groups, a connectivity matrix is first generated for a region-of-interest (ROI) set for each subject from their resting state fMRI data. This all-to-all connectivity matrix is reduced to only include significant connections by applying a threshold on the minimum weight for edges included in the network. This new topology undergoes eigenvector-based community designation, where the communities are ranked by their influence over the network. This ranking of nodes based on their community's influence is used to train neural networks to recognise whether a subject is healthy or has amnestic mild cognitive impairment (aMCI) or Alzheimer's disease (AD). To understand the changes required to convert a subject from one condition to another, the influence of their nodes are altered so that the neural network would change their classification to an alternative state. This alteration, to achieve this change in classification, captures the difference in the influence of ROIs when comparing between different neurological conditions.

\subsection{Dataset}

The data used here was from the 'Resting-state fMRI in Dementia Patients' dataset [13], obtained from the Harvard Dataverse database. The MRI data was obtained using a Siemens 3T MRI system (Magnetom Allegra, Siemens, Erlangen, Germany) for ten patients with a probable AD diagnosis (by NINCDSADRDA consensus criteria [14]), 10 aMCI patients [15] and 10 healthy elderly subjects $(\mathrm{HC})$.

The subjects underwent a resting state EPI fMRI scan $(\mathrm{TR}=2080 \mathrm{~ms}$, TE $=30 \mathrm{~ms}$, 32 axial slices parallel to AC-PC plane, matrix $64 \times 64$, in plane resolution $3 x 3 \mathrm{~mm}^{2}$, slice thickness $=2.5 \mathrm{~mm}, 50 \%$ skip, flip angle 70 degrees). The duration of the scan was 7 minutes and 20 seconds, yielding 220 volumes. Subjects were instructed to keep their eyes closed throughout, refrain from thinking of anything in particular and to avoid falling asleep. An anatomical T1-weighted three dimensional MDEFT (modified driven equilibrium Fourier transform) scan was also acquired for each subject $(\mathrm{TR}=1338 \mathrm{~ms}, \mathrm{TE}=2.4 \mathrm{~ms}, \mathrm{TI}=910 \mathrm{~ms}$, flip angle $=15$ degrees, matrix $=256 \times 224 \times 176, \mathrm{FOV}=256 x 224 \mathrm{~mm}^{2}$, slice thickness $=1 \mathrm{~mm}$, total scan time $=12 \mathrm{~min}$ ).

\subsection{Preprocessing}

The functional data was preprocessed using the CONN toolbox (CONN: functional connectivity toolbox, [16]) for SPM12 (www.fil.ion.ucl.ac.uk/spm) and MATLAB version 2018a. 
Spatial Preprocessing The first four volumes of the functional scans were removed in order to eliminate saturation effects and to allow the signal to stabilise. Functional data were slice-time adjusted and corrected for motion. The high resolution $\mathrm{T} 1$ weighted anatomical images were coregistered with the mean EPI image. They were segmented into grey matter (GM), white matter (WM) and cerebrospinal fluid (CSF) masks and were spatially normalised to the Montreal Neurological Institute (MNI) space [17]. The obtained transformation parameters were applied to the motion corrected functional data, and an $8 \mathrm{~mm}$ FWHM Gaussian kernel was applied for spatial smoothing. It should be noted that the use of spatial smoothing on fMRI data can affect the properties of functional brain networks, including a possible overemphasis of strong, shortrange links, changes in the identities of hubs of the network, and decreased intersubject variation [18].

Temporal Filtering In order to mitigate physiological and movement-related noise, the aCompCor technique was used. aCompCor identifies and removes the first five principal components of the signal from the CSF and WM masks (eigenvectors of the PCA decomposition of the EPI timecourse averaged over the CSF and WM), as well as the motion parameters, their first-order temporal derivatives and a linear detrending term [19]. One subject's scan was excluded from the analysis due to excessive motion. Scrubbing and motion regression were also performed. The preprocessed functional data were then bandpass filtered $(0.008 \mathrm{~Hz}<f<0.09 \mathrm{~Hz})$ using a fast Fourier transform (FFT).

\subsection{Connectivity Matrix Generation}

One hundred and thirty-two (132) ROIs were defined by the default CONN atlas which combines the FSL Harvard-Oxford cortical and subcortical areas and the AAL atlas cerebellar areas. Connectivity between the 132 ROIs was assessed for the 7-minute resting state scan for each subject. We constructed $132 \times 132$ ROIto-ROI correlation (RRC) matrices of Fisher z-transformed bivariate correlation coefficients (Pearsons r) using the ROIs described above. For each subject, a graph adjacency matrix $A(i, j)$ was computed by thresholding the RRC matrix r(i,j) using a Cluster-Span Threshold (CST [20]).

\subsection{Cluster-Span Threshold}

An unbiased Cluster-Span threshold (CST) [20] was used in generating the adjacency matrix. CST is especially suitable as it performs well in distinguishing functional connectivity between $\mathrm{HC}$ and $\mathrm{AD}$ subjects [21]. The threshold generates a topology that excludes edges with weights smaller than the chosen value. CST is selected so that the topology generated contains the same number of clustered triples and spanning triples. 


\subsection{Communities of Dynamical Influence}

The network is assigned into Communities of Dynamical Influence (CDI) based on the connections and influence of nodes in the network. CDI are defined in [5] where community designation is achieved by using multiple (often three) eigenvectors to define a coordinate system. The nodes, which are further from the origin of this system than any of their connections, are defined as leaders of separate communities. Each of these communities is populated with other nodes that lie on a path that connects to the leader node of that community. Each node is assigned to only one community, the community is chosen by assessing which leader is most closely aligned to that node. This alignment is assessed by comparing the position vector, from the origin of the coordinate system, for the leader nodes and the node to be assigned. The dot product of these position vectors determines the leader that is best aligned to the node.

Once community designation is complete, the order of influence is determined by evaluating the largest entry of the most dominant eigenvector for each community. The dominant eigenvector of the connectivity matrix is known to be a nonnegative vector. The community that contains the node with the largest $\mathbf{v}_{1}$ value is ranked as the most influential community, with the other communities ranked in descending order according to their largest $\mathbf{v}_{1}$. For each subject, a vector is produced that denotes the ranking of the community each node is in. This vector shall be referred to as the Influence Vector with values assigned to ROIs between 0 and 1 , where these extremes mark the least and most influential community respectively.

In this paper, CDI is determined from the three most dominant eigenvectors of the undirected connectivity matrix after applying the CST. These are the eigenvectors associated with the largest eigenvalues in magnitude and are shown in [5] to identify the nodes that are most effective at driving the network to consensus.

\subsection{Pattern Recognition}

A neural network (NN) is employed to recognise patterns in the influence vector and associate these patterns with the subject status of healthy control (HC) or aMCI or AD. A two-layer, feed-forward, neural network was used since this simple architecture was shown to be capable of capturing sufficient information from the influence vector to produce accurate classifications. The NN employed sigmoid output neurons, scaled conjugate gradient backpropagation and 10 hidden neurons [22]. Each input vector, $\mathbf{x}$, is scaled to fit in the range $[-1,1]$ and the performance is evaluated using cross-entropy [23], with the cost function, $c$, a mean of the individual values,

$$
c=-\frac{1}{n} \sum_{i=1}^{x} y \ln (a)
$$

where $n$ is the total number of items of data in a set of inputs $x$, with the network output $a$ and the desired output $y$. 
To identify and reduce variance when using a small data set, five separate neural networks were trained with different compositions of training and validation sets. No test set was used due to the small size of the data set and the fact that the risk of over-fitting is not a major concern in the intended application. For a neural network to be trained successfully the cross-entropy cost function had to be below $c=0.1$ with all networks reporting $100 \%$ accuracy from their confusion matrices. The five neural networks were trained on 29 subjects with the training sets varied between 21 and 25 subjects, where the validation set contained the remaining subjects and was subjects from all three of the classifications. Below 21 there was insufficient training data to effectively train the network. The mean of the five NN outputs was assessed and in Fig. 1 the variance between different NNs is also reported.

\subsection{Optimisation of the Influence Vector}

The neural network outputs a three element vector, $a$, with non-negative entries that represents the three possible subject conditions, where $\sum_{j=1}^{3} a_{j}=1$ and the largest element indicates the condition selected by the network. An optimiser, using a sequential quadratic programming method [24], is employed to alter the input vector so that the neural network identifies it as having a different condition. The optimiser aims to maximise $a_{j}$ where $j$ represents the target condition. The alteration to the input vector is recorded to identify the ROIs that were altered to change a subject from their current condition to another state.

\section{$3 \quad$ Results}

A large variability between subjects is observed, even when they are represented by their ROI community influence. For example, Table 1 shows that for each classification most of the ROIs are, for at least one of the subjects, placed in both the most and the least influential communities (top and bottom CDI respectively). Despite this variability, trends emerge when comparing the different classification in Table 1 with the percentage of ROIs displaying either an upward or downward trend from $\mathrm{HC}$, through aMCI to $\mathrm{AD}$ in each row. One of the most notable results is that, for $\mathrm{AD}, 43 \%$ of the ROIs present in the top CDI, for at least one subject, were not in the bottom CDI for any other subject. Whilst only $53 \%$ of the 132 ROIs were present in the top CDI for at least a single AD subject. This means that around $81 \%$ of the ROIs, in the most influential community for at least one AD subject, are not in the least influential community for any other AD subject. This is a far greater consistency than seen from the aMCI $(\sim 29 \%)$ and HC $(\sim 15 \%)$ subjects. To a less significant extent the HC subjects present the inverse pattern, whereby they have the highest number of ROIs that are present in the bottom CDI and not included in the top CDI for any other HC subject (38\%), compared to aMCI (24\%) and AD (14\%). 
Table 1: The number of ROIs that are included in the most (top) and least (bottom) influential communities of dynamical influence (CDI) are shown alongside the number of ROIs that are in the top community for at least one subject but not the bottom for any others and vice versa.

\begin{tabular}{r|ccc}
\multirow{2}{*}{} & \multicolumn{3}{|c}{ \% of ROIs } \\
\cline { 2 - 4 } Present in bottom CDI & 71 & 79 & 84 \\
\hline Present in bottom CDI \& not in top CDI & 27 & 19 & 12 \\
Present in top CDI & 85 & 77 & 53 \\
Present in top CDI \& not in bottom CDI & 13 & 22 & 43
\end{tabular}

\subsection{Altering Influence in Healthy Control Subjects}

A mean alteration was produced from alterations generated using five separate neural networks, as described in the Methods section. Three different conversions are detailed here; Ten HC subjects were converted to the classification of aMCI, ten $\mathrm{HC}$ subjects were also converted to $\mathrm{AD}$, and finally ten aMCI subjects were converted to the classification of $\mathrm{AD}$. The results of these conversions highlight the ROIs that undergo the greatest changes in connectivity and influence when a person is affected by MCI and/or AD, see Fig. 1 (a), (b) \& (c). These three conversions were also investigated in the other direction, eg. converting from $\mathrm{AD}$ to $\mathrm{HC}$, with the results producing similar findings to those detailed herein and so not reported separately.

Parahippocampal Gyri. The hippocampus and parahippocampal regions are well known to have a significant role in memory formation [3], which is an area that notably declines in those with aMCI and AD. In [25] the parahippocampal gyri were noted to decrease in functional connectivity in AD subjects when compared with aMCI subjects. Our results support these findings for the right PHG, where it loses significant influence in the conversion from $\mathrm{HC}$ to $\mathrm{AD}$ and from aMCI to $\mathrm{AD}$, in Fig. 1 (d). The anterior and posterior divisions of the right PHG reporting some of the largest alterations in these conversion (see Fig. 1 (a) and Fig. 1 (c) respectively). Interestingly in [3], subjects with greater clinical impairment were found to rely more on their right parahippocampal gyrus (PHG). Those with a reliance on the right PHG were also those whom declined the most over 2.5 years of clinical follow-up. It was hypothesised that this extra reliance on the right PHG could be a marker for impending clinical decline. This compensatory mechanism appears to be visible in the results of Fig. 1 (d) where the anterior division of the right PHG gains influence when converting $\mathrm{HC}$ subjects to aMCI with a mean alteration z-score of 0.83 .

The behaviour of the hippocampus mirrors that of the parahippocampal regions in the $\mathrm{HC}$ to $\mathrm{AD}$ conversions. The right hippocampus loses a significant amount of influence (z-score of -1.18) while the left side gains some influence 


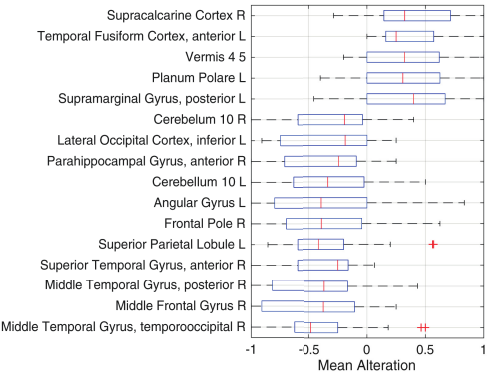

(a)

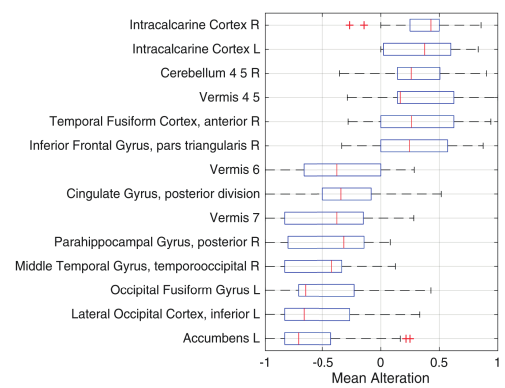

(c)

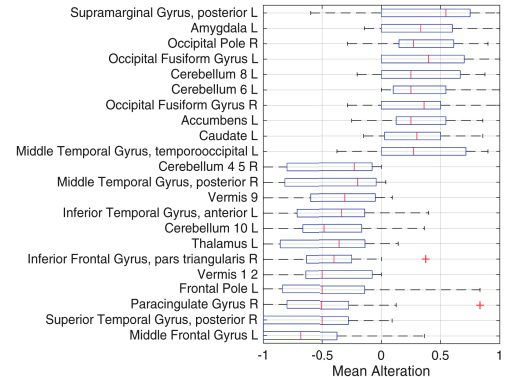

(b)

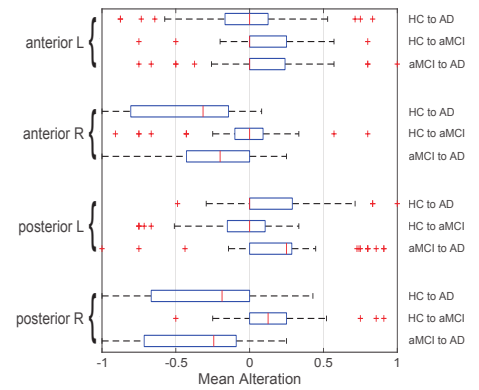

(d)

Fig. 1: The largest alterations of ROI influence $( \pm 1.5 \mathrm{z}$-score $)$ required to change the classification of (a) $10 \mathrm{HC}$ subjects to $\mathrm{AD}$, (b) $10 \mathrm{HC}$ subjects to aMCI, and (c) 10 aMCI subjects to AD. In (d) the alterations to the parahippocampal regions are reported for each conversion. A positive alteration indicates that a ROI's influence has increased. The mean alteration is assessed from five conversions using different neural networks. The median, $25^{\text {th }}$ and $75^{\text {th }}$ percentile are detailed with the whiskers extending to the most extreme data points. Outliers lie more than three scaled median absolute deviations away from the median and are excluded.

(z-score of 0.41). This finding is supported by [26] where disrupted connectivity between the right hippocampus and several brain regions was seen for $\mathrm{AD}$ subjects, whilst connectivity between the left hippocampus and the prefrontal cortex was relatively increased. In [27] a more extensive connectivity disruption was found in both sides of the hippocampus, where the contrast in results to [26] was attributed to greater severity of AD in subjects studied in [27] with hippocampal connectivity thought to decline progressively during the disease.

Default Mode Network. Two prominent regions in the default mode network $(\mathrm{DMN})$, the posterior cingulate cortex and the precuneus cortex, have frequently been identified as early markers in AD [28]. Compared to controls, AD patients 
have been shown to exhibit lower connectivity in the precuneus and posterior cingulate cortex within the DMN [29].

Our findings support [29] where the posterior cingulate cortex loses influence in the $\mathrm{HC}$ to $\mathrm{AD}$ case with a $\mathrm{z}$-score of -0.96 , while in the $\mathrm{HC}$ to aMCI case it gains some influence with a z-score of 0.49 . It is therefore not surprising that this region loses significant influence in the aMCI to AD conversion in Fig. 1 (c) with a z-score of -1.53. In the conversions from $\mathrm{HC}$ to $\mathrm{AD}$ and from $\mathrm{HC}$ to aMCI, the precuneus cortex becomes notably less influential, indicated by a negative mean alteration with a $\mathrm{z}$ score of -1.14 and -1.00 , respectively. These results indicate that both ROIs are clear indicators of $\mathrm{AD}$, but this analysis suggests their decline appears to occur at different stages of the disease with results suggesting that the precuneus cortex declines earlier as depicted in Fig. 2.

Furthermore, a network comparison in [30] between controls and AD highlighted a decrease in functional connectivity between the DMN and posterior cingulate gyrus, precuneal cortex, and lateral occipital cortex (LOC). Our results support this finding where, in Fig. 1 (a), the left inferior division of the LOC loses significant influence in the $\mathrm{HC}$ to $\mathrm{AD}$ conversion (z-score of -1.53) while the right side only changes slightly (z-score of -0.16$)$. An even more significant swing in influence is seen, in Fig. 1 (b), for the LOC's left inferior division in the aMCI to AD conversion (z-score of -2.28). This ROI presents a similar pattern to the posterior cingulate cortex by gaining influence in the $\mathrm{HC}$ to aMCI conversion (z-score of 0.99 ), but losing influence in both conversions to AD. It is also worth noting that the superior divisions of the LOC presents the opposite changes in influence but to a less significant degree than the left inferior division.

Other key DMN ROIs are seen to lose influence in the conversions, with the left Angular gyrus and middle frontal gyri displaying prominently in Fig. 1 (a) and (b).

\subsection{Calcarine.}

The calcarine, sensorimotor and anterior cingulate regions have been shown to be spared significant damage until the late stages of AD [31]. Our results indicate that by avoiding significant damage the intracalcarine and supracalcarine regions gain greater influence. The right supracalcarine cortex tops the positive alterations in Fig. 1 (a) and both sides of the intracalcarine cortex top the positive alterations in Fig. 1 (c). However, the bi-lateral intracalcarine cortex and the left supracalcarine cortex lose influence when converting from HC to MCI, so avoiding damage might not provide the whole picture for why these calcarine regions become more influential in the $\mathrm{HC}$ to $\mathrm{AD}$ conversion.

\section{Conclusions}

This paper presents a new approach to understanding the impact of changes in brain region connectivity, which are brought about by neurological conditions. Brain region assignment to influence ranked communities reveals that those with 
(a)

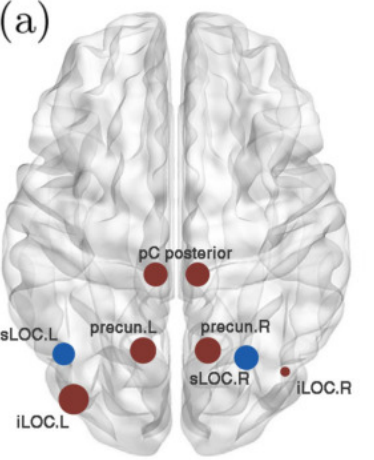

(b)

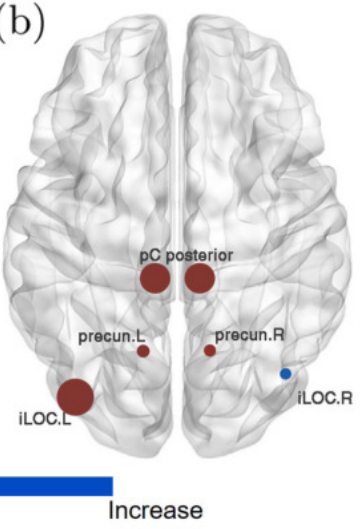

Fig. 2: Superior view of mean alterations to influence of default mode network and lateral occipital cortex (LOC). Circle size proportional to z-score for (a) HC to $\mathrm{AD}$ and (b) aMCI to $\mathrm{AD}$ conversion. ROIs shown: posterior cingulate (pC), Precuneus (Precun), inferior and superior LOC (iLOC \& sLOC) with L and R indicating left and right respectively.

AD have a significantly higher degree of commonality in their most influentially connected regions, when compared with $\mathrm{HC}$ and aMCI subjects. The communities identifies as influential therefore appear to be shared to a greater extent in AD subjects, compared to the aMCI and HC.

The detection of brain region influence enables the classification of conditions and captures the patterns of functional changes that lead to aMCI and AD. The right parahippocampal gyrus (PHG) is confirmed as playing a key role in the decline to AD. In particular, the results supported findings of compensatory activity in this region, where it was seen to maintain, if not gain, some influence in the conversion from HC to aMCI. The right PHG then experienced a significant decline for the conversion to an AD classification. The results supported previous findings on the importance of the default mode network (DMN) in the development of aMCI and AD. Additionally, the posterior cingulate gyrus and the lateral occipital cortex are noted as promising indicators of future conversions from aMCI to AD. Finally, the calcarine is confirmed as a region that defies decline in those with AD. Interestingly, the intra- and supracalcarine regions are seen to decline for those with aMCI but their influence then increases for subjects with AD.

\section{References}

1. Dawe, B., Procter, A. and Philpot, M., 1992. Concepts of mild memory impairment in the elderly and their relationship to dementiaa review. International Journal of Geriatric Psychiatry, 7(7), pp.473-479. 
2. Apostolova, L.G., Dutton, R.A., Dinov, I.D., Hayashi, K.M., Toga, A.W., Cummings, J.L. and Thompson, P.M., 2006. Conversion of mild cognitive impairment to Alzheimer disease predicted by hippocampal atrophy maps. Archives of neurology, 63(5), pp.693-699.

3. Dickerson, B.C., Salat, D.H., Bates, J.F., Atiya, M., Killiany, R.J., Greve, D.N., Dale, A.M., Stern, C.E., Blacker, D., Albert, M.S., and Sperling, R.A.: Medial temporal lobe function and structure in mild cognitive impairment. Annals of neurology, 56(1), pp. 27-35. (2004). doi:10.1002/ana.20163

4. Friston, K.J.: Functional and effective connectivity in neuroimaging: A synthesis. Hum Brain Mapp 2, pp. 56-78. (1994) doi:10.1002/hbm.460020107

5. Clark, R., Punzo, G., and Macdonald, M.: Network Communities of Dynamical Influence. arXiv. (2019). arXiv:1908.10129

6. Varshney LR, Chen BL, Paniagua E, Hall DH, Chklovskii DB. Structural properties of the Caenorhabditis elegans neuronal network. PLoS computational biology. 2011;7(2):e1001066.

7. Shi, J. and Malik, J., 2000. Normalized cuts and image segmentation. Departmental Papers (CIS), p.107.

8. Suk, H.I., Lee, S.W., Shen, D. and Alzheimers Disease Neuroimaging Initiative, 2015. Latent feature representation with stacked auto-encoder for AD/MCI diagnosis. Brain Structure and Function, 220(2), pp.841-859.

9. Hojjati, S.H., Ebrahimzadeh, A., Khazaee, A., Babajani-Feremi, A. and Alzheimers Disease Neuroimaging Initiative, 2017. Predicting conversion from MCI to AD using resting-state fMRI, graph theoretical approach and SVM. Journal of neuroscience methods, 282, pp.69-80.

10. Khazaee, A., Ebrahimzadeh, A. and Babajani-Feremi, A., 2016. Application of advanced machine learning methods on resting-state fMRI network for identification of mild cognitive impairment and Alzheimers disease. Brain imaging and behavior, 10(3), pp.799-817.

11. Khazaee, A., Ebrahimzadeh, A., Babajani-Feremi, A. and Alzheimers Disease Neuroimaging Initiative, 2017. Classification of patients with MCI and AD from healthy controls using directed graph measures of resting-state fMRI. Behavioural brain research, 322, pp.339-350.

12. Forouzannezhad, P., Abbaspour, A., Fang, C., Cabrerizo, M., Loewenstein, D., Duara, R., Adjouadi, A.: A survey on applications and analysis methods of functional magnetic resonance imaging for Alzheimers disease. J Neurosci Methods 317, pp. 121-140. (2019). doi:10.1016/j.jneumeth.2018.12.012

13. Mascali, D., DiNuzzo, M., Gili, T., Moraschi, M., Fratini, M., Maraviglia, B., Serra, L., Bozzali, and M., Giove, F.: Resting-state fMRI in dementia patients. (2015) Harvard Dataverse. doi.org/10.7910/DVN/29352

14. McKhann, G., Drachman, D., Folstein, M., Katzman, R., Price, D., and Stadlan, E.M.: Clinical diagnosis of Alzheimers disease: report of the NINCDS-ADRDA Work Group under the auspices of Department of Health and Human Services Task Force on Alzheimers Disease. Neurology 34, pp. 939-944. (1984) doi:10.1212/wnl.34.7.939

15. Petersen, R.C., Doody, R., Kurz, A., Mohs, R.C., Morris, J.C., Rabins, P.V., Ritchie, K., Rossor, M., Thal, L., and Winblad, B.: Current concepts in mild cognitive impairment. Arch. Neurol. 58, pp. 1985-1992. (2001) doi:10.1001/archneur.58. 12.1985

16. Whitfield-Gabrieli, S., and Nieto-Castanon, A.: Conn: a functional connectivity toolbox for correlated and anticorrelated brain networks. Brain Connect. 2, pp. 125-141. (2012) doi:10.1089/brain.2012.0073 
17. Ashburner, J., and Friston, K.J.: Unified segmentation. NeuroImage 26, pp. 839851. (2005). doi:10.1016/j.neuroimage.2005.02.018

18. Alakörkkö, T., Saarimäki, H., Glerean, E., et al. Effects of spatial smoothing on functional brain networks. European Journal of Neuroscience. 2017;46(9):2471-2480.

19. Behzadi, Y., Restom, K., Liau, J., and Liu, T.T.: A component based noise correction method (CompCor) for BOLD and perfusion based fMRI. NeuroImage 37, pp. 90-101. (2007) doi.org/10.1016/j.neuroimage.2007.04.042

20. Smith, K., Azami, H., Parra, M. A., Starr, J. M., and Escudero, J.: Cluster-span threshold: An unbiased threshold for binarising weighted complete networks in functional connectivity analysis. 2015 37th Annual International Conference of the IEEE Engineering in Medicine and Biology Society (EMBC) 147, pp. 2840-2843. IEEE (2015). doi:10.1109/EMBC.2015.7318983

21. Smith, K., Abasolo, D. and Escudero, J., 2016, August. A comparison of the clusterspan threshold and the union of shortest paths as objective thresholds of EEG functional connectivity networks from Beta activity in Alzheimer's disease. In 2016 38th Annual International Conference of the IEEE Engineering in Medicine and Biology Society (EMBC) (pp. 2826-2829). IEEE.

22. Mathworks. (2019). nprtool: Neural Net Pattern Recognition tool (r2019a). Retrieved August 16, 2019 from https://uk.mathworks.com/help/deeplearning/ref/ nprtool.html

23. Mathworks. (2019). crossentropy: Neural Network performance (r2019a). Retrieved August 16, 2019 from https://uk.mathworks.com/help/deeplearning/ref/ crossentropy.html

24. Mathworks. (2019). fminunc Unconstrained Minimization (r2019a). Retrieved August 16, 2019 from http://uk.mathworks.com/help/optim/ug/ fminunc-unconstrained-minimization.html

25. Gili, T., Cercignani, M., Serra, L., Perri, R., Giove, F., Maraviglia, B., Caltagirone, C. and Bozzali, M., 2011. Regional brain atrophy and functional disconnection across Alzheimer's disease evolution. Journal of Neurology, Neurosurgery \& Psychiatry, 82(1), pp. 58-66. (2011). doi:10.1136/jnnp.2009.199935

26. Wang, L., Zang, Y., He, Y., Liang, M., Zhang, X., Tian, L., Wu, T., Jiang, T. and Li, K., 2006. Changes in hippocampal connectivity in the early stages of Alzheimer's disease: evidence from resting state fMRI. Neuroimage, 31(2), pp.496-504.

27. Allen G, Barnard H, McColl R, et al. Reduced Hippocampal Functional Connectivity in Alzheimer Disease. Arch Neurol. 2007;64(10):1482-1487. doi:10.1001/ archneur.64.10.1482

28. Rombouts, S.A.R.B., Barkhof, F., Goekoop, R., Stam, C.J., and Scheltens, P.: Altered resting state networks in mild cognitive impairment and mild Alzheimer's disease: an fMRI study. Human brain mapping. 26(4), pp. 231-239. (2005). doi: $10.1002 / \mathrm{hbm} .20160$

29. Binnewijzend, M.A., Schoonheim, M.M., Sanz-Arigita, E., Wink, A.M., van der Flier, W.M., Tolboom, N., Adriaanse, S.M., Damoiseaux, J.S., Scheltens, P., van Berckel, B.N., Barkhof, F.: Resting-state fMRI changes in Alzheimer's disease and mild cognitive impairment. Neurobiology of Aging, Volume 33, Issue 9, pp. 2018 2028. (2012) https://doi.org/10.1016/j.neurobiolaging.2011.07.003

30. Hafkemeijer, A., Mller, C., Dopper, E.G., Jiskoot, L.C., Schouten, T.M., van Swieten, J.C., van der Flier, W.M., Vrenken, H., Pijnenburg, Y.A., Barkhof, F. and Scheltens, P., 2015. Resting state functional connectivity differences between behavioral variant frontotemporal dementia and Alzheimers disease. Frontiers in human neuroscience, 9, p.474. doi:10.3389/fnhum.2015.00474 
31. Brun, A. and Englund, E., 1981. Regional pattern of degeneration in Alzheimer's disease: neuronal loss and histopathological grading. Histopathology, 5(5), pp.549564. 